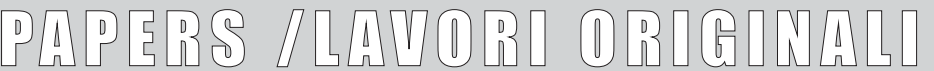

\section{Controlli ambientali sulla diffusione di sierotipi di Salmonella spp in acque fluviali}

\author{
Lucia Bonadonna', Emma Filetici², Assunta Nusca', Rosa Paradiso' \\ Istituto Superiore di Sanità \\ 'Dipartimento Ambiente e Connessa Prevenzione Primaria \\ ${ }^{2}$ Dipartimento Malattie infettive, Parassitarie ed Immunomediate
}

Key words: genus Salmonella, serotypes, water

Environmental investigations on Serotypes of Salmonella spp. in rivers

\section{SUMMARY}

Data on serotypes of Salmonella isolates from rivers are presented. The microrganism was detected in high concentrations in all the sampling sites. These values show its regular occurrence in the examined waters all the time, even if a seasonal trend was observed with higher levels in summer and fall. Salmonella typhimurium and $S$. infantis were the prevalent serotypes. These serotypes are also among those more prevailing in ltaly as it is pointed out by the surveillance network of gastrointestinal infections.

\section{INTRODUZIONE}

In Europa, negli anni più recenti, l'incidenza delle salmonellosi si è sostanzialmente ridotta: il numero totale di patologie nell'uomo ha avuto una flessione intorno al $31 \%$ (11). La situazione varia tuttavia nei diversi Paesi europei in rapporto all'attività dei programmi di controllo che si basa, innanzitutto, sulla sorveglianza epidemiologica delle salmonellosi trasmesse attraverso gli alimenti e sul riconoscimento degli episodi epidemici a livello nazionale e/o internazionale (12). Sulla base dei piani di vigilanza viene svolta anche attività di monitoraggio in habitat extrabiologici; vengono quindi effettuate verifiche ambientali per tenere sotto controllo quell'ambito meno percepibile del ciclo di diffusione del patogeno che è rappresentato dall'ambiente, e dalle acque in particolare, ma che comunque contribuisce al suo completamento.

In Italia sono attive due reti di sorveglianza delle infezioni da Salmonella: la rete Enter-net, attiva dal 1994, in cui confluiscono i dati degli isolamenti dall'uomo e la rete Enter-vet, attiva dal 2002, che raccoglie dati da campioni di origine veterinaria $(6,13)$. In particolare, nell'ambito della rete Enter-net, la ricerca delle salmonelle nell'ambiente è generalmente effettuata dalle Agenzie Regionali di Prevenzione Ambientale, ma non tutte le regioni hanno dati aggiornati, anche a causa delle differenti competenze sviluppate. L'Istituto Superiore di Sanità, infine, coordina a livello nazionale la rete Enter-net Italia ed invia i dati alla rete europea di sorveglianza delle infezioni da Salmonella che ha sede presso la
Health Protection Agency in Gran Bretagna.

I microrganismi appartenenti al genere Salmonella sono batteri ubiquitari di origine enterica. La classificazione, più volte rielaborata, deriva da quelle di Kauffmann-White e da quella di Le Minor ed è soggetta ad aggiornamenti annuali $(5,19)$.

Il genere, distinto in due sole specie, $S$. enterica e $S$. bongori, comprende sierotipi caratterizzati in base alla diversa composizione di antigeni somatici e flagellari e, in alcuni casi, è anche funzione di specifici caratteri biochimici. Della specie enterica, suddivisa in sei sottospecie, si conoscono più di 2400 sierotipi.

Le salmonelle rappresentano uno dei più comuni agenti eziologici di enteriti a trasmissione fecaleorale. In Italia, circa 20 decessi/anno sono associati a salmonellosi, generalmente segnalati in soggetti di età superiore ai 55 anni (15).

L'ambiente rappresenta un serbatoio di Salmonella, così come di un gran numero di microrganismi autoctoni ed alloctoni. Le salmonelle hanno come habitat il tratto intestinale dell'uomo e degli animali e la loro diffusione nell'ambiente è conseguente a contaminazione di origine fecale. Inoltre, serbatoi di infezione sono gli avicoli (polli e tacchini in particolare) ed i suini, anche se, non bisogna dimenticare, che anche gli animali da compagnia (cani, gatti, rettili) possono albergare ed eliminare salmonella $(4,6,20)$.

Nell'ambiente, nonostante condizioni di certo sfavorevoli, legate a fattori di variabilità climatica, di carenza di nutrienti e di competizione biologica, molti fattori giocano un ruolo non trascurabile per la sopravvivenza e la diffusione del microrgani- 
smo (16). Salmonella viene sempre rilevata nelle acque reflue e nei fanghi di depurazione e nessun corpo idrico superficiale è esente dalla sua presenza (3). I corsi d'acqua assumono quindi un significato rilevante sia nell'ecologia ambientale sia dal punto di vista igienico-sanitario se si considerano le diverse destinazioni d'uso dell'acqua: approvvigionamento a scopo potabile, uso ricreativo ed irriguo. In quest'ultimo caso, infatti, l'utilizzo di acque inquinate per uso agricolo rappresenta una fonte diretta di contaminazione ed un rischio per la salute umana, soprattutto quando vengono irrigati vegetali da consumare crudi (17). Lo stato di criticità delle risorse idriche e le potenziali condizioni di rischio sanitario che ne derivano diventano quindi fattori di interesse prioritario in un ambito di prevenzione e tutela della salute e di protezione dell'ambiente. L'impostazione di piani di sorveglianza e di programmi di gestione delle risorse a garanzia della salute delle popolazioni e della salvaguardia dell'ambiente richiedono pertanto l'acquisizione di informazioni sempre più ampie e approfondite.

$\mathrm{E}$ in questo ambito che si inserisce il lavoro di seguito presentato che vuole fornire un contributo conoscitivo sulle concentrazioni di Salmonella e sulla diffusione dei vari sierotipi in acque superficiali di un'area della Regione Campania dove limitata è la sorveglianza ambientale diretta alla determinazione del microrganismo nelle acque. Lo studio fa parte in un più ampio programma di controlli ambientali/sanitari in applicazione dell' articolo 6 della Legge 305/1989 e il territorio interessato, dal 1992, è stato dichiarato "area ad elevato rischio di crisi ambientale". Il sistema di sorveglianza, ancora in atto, attraverso l'individuazione delle specifiche e puntuali cause di degrado e lo sviluppo di piani di risanamento, mira alla riduzione del livello di rischio sanitario esistente in un ambito di tutela e prevenzione per la salute delle popolazioni e di rivalutazione del territorio.

\section{MATERIALI E METODI}

\section{Siti di prelievo}

I prelievi sono stati effettuati in otto stazioni fluviali situate sul bacino idrografico del fiume Sarno (figura I). Numerose industrie conciarie e conserviere gravitano sull'area e riversano i loro effluenti nei corpi idrici che ricevono anche scarichi civili scarsamente trattati e/o non controllati. Difficoltà di gestione delle risorse, cattiva gestione dei rifiuti, tendenza alla polarizzazione degli insediamenti produttivi ed elevata densità demografica dell'area contribuiscono a creare disfunzioni dei sistemi di depurazione dei reflui. La sinergia dei molteplici fattori associati concorre quindi ad alterare la qualità dell'intero sistema fluviale che, con l'immissione nel Golfo di Napoli, influenza anche gli ecosistemi marini e le attività turistiche esistenti lungo il litorale.

\section{Determinazioni analitiche.}

Nel corso di due anni di indagine, sono stati analizzati oltre 300 campioni di acqua prelevati con cadenza mensile. Prima dell'inizio della ricerca sono stati esperiti sopralluoghi che sono proseguiti nel corso dello studio per verificare l'eventuale evoluzione dello stato ambientale delle stazioni di prelievo selezionate come più significative per l'ottenimento dei risultati. Tutte le operazioni di prelievo e di trasporto dei campioni sono state svolte seguendo le specifiche norme tecniche (1). I campioni d'acqua sono stati trasportati in laboratorio in contenitori refrigerati ed analizzati entro le 24 ore dal prelievo. Il metodo di seguito descritto è stato utilizzato per la determinazione quantitativa di Salmonella spp.

Dopo filtrazione di appropriati volumi d'acqua, l'analisi è stata eseguita seguendo la procedura che prevede le fasi di prearricchimento (membrana in $200 \mathrm{ml}$ di Acqua Peptonata Tamponata a $37^{\circ} \mathrm{C}$ per 24 ore), arricchimento (diluizioni scalari della brodocoltura inoculate in tubi di Brodo Rappaport Vassiliadis incubando a $42^{\circ} \mathrm{C}$ per 24 ore), isolamento su terreno colturale selettivo (dai tubi di arricchimento su Hektoen Enteric Agar incubando a $36^{\circ} \mathrm{C}$ per $24+24$ ore). È stata quindi registrata la crescita o l'assenza di crescita di colonie tipiche di colore verde con margini netti con o senza centro nero in funzione delle diluizioni scalari della brodocoltura di provenienza. I risultati sono stati quindi calcolati come valore di concentrazione dell'indice MPN/100 ml di campione di acqua (Most Probable Number). Un elevato numero di colonie di sospette salmonelle sono state poi isolate per la verifica dell'appartenenza al genere, utilizzando test miniaturizzati di prove biochimiche (API 20E, bioMérieux). A queste determinazioni è seguita la tipizzazione sierologica mediante agglutinazione con metodica rapida su vetrino con antisieri specifici (Statens Serum Institut, Biogenetics).

\section{RISULTATI}

In figura II sono riportati i valori medi di Salmonella calcolati nel corso dei due anni di indagine.

Durante il primo anno di studio, a concentrazioni elevate del microrganismo, variabili tra $10^{2}$ MPN/100 $\mathrm{ml}$ e più di $10^{4} \mathrm{MPN} / 100 \mathrm{ml}$ in funzione dei siti di prelievo, corrispondevano basse oscillazioni nelle densità degli isolati in ciascuna stazione di campionamento. Nel secondo anno le concentrazioni di Salmonella hanno mostrato un apprezzabile decremento che, a fronte delle den- 
sità osservate nell'anno precedente, si sono ridotte con valori di poco superiori a $10^{3} \mathrm{MPN} / 100 \mathrm{ml}$. Ciò nonostante, in questo caso, sono state osservate maggiori variabilità nel numero degli isolati in ciascuna stazione, oscillazioni da attribuire a specifiche condizioni meteorologiche locali.

Come tendenza generale è stato osservato che le concentrazioni più elevate erano riscontrate nel periodo estivo-autunnale. È comunque da considerare che le più alte densità di Salmonella erano più che altro associate a campioni prelevati in corrispondenza di aree con maggiore densità abitativa (stazioni di prelievo 6-8) e, di conseguenza, è ipotizzabile, come esito di un maggiore apporto di carico microbico da reflui civili.

$\mathrm{Nel}$ corso dell'indagine sono stati selezionati 95 ceppi di Salmonella spp e sono stati identificati 45 sierotipi diversi; la caratterizzazione sierologica degli isolati ha messo in evidenza che i sierotipi più ricorrenti - $S$. Typhimurium $(13.7 \%)$, $S$. sottospecie II (8.4\%), S. Infantis (5.3\%), S. Bredeney, S. Stanley e $S$. Derby (4.2\%) - sono stati ritrovati con notevole regolarità in corrispondenza di tutte le stazioni di prelievo (tabella 1). Con una frequenza più ridotta $(3.2 \%)$ sono state rilevate $S$. Farsta, S. Kottbus, S. Lagos e S. Rissen.

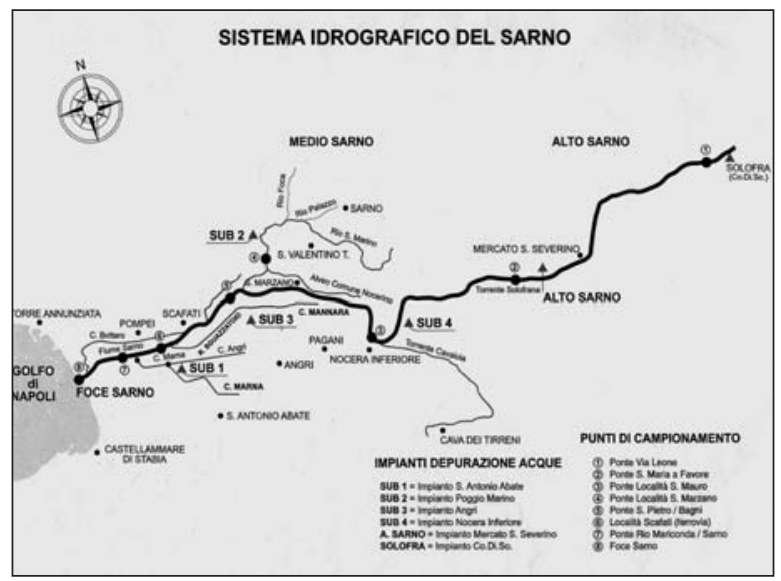

Figura I. Bacino idrografico del fiume Sarno. I numeri da I a 8 indicano le stazioni di prelievo considerate nello studio.

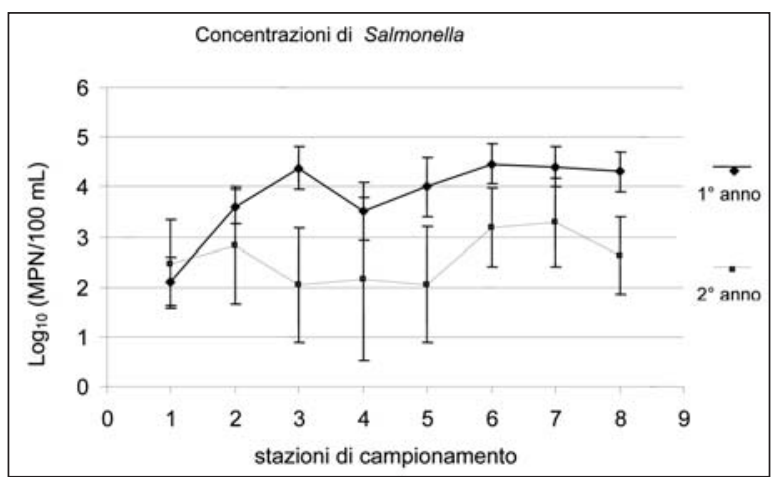

Figura II. Valori medi di Salmonella calcolati nelle acque nel corso dei due anni di indagine.
Tabella I. Sierotipi di Salmonella identificati, loro frequenza e stazioni di isolamento.

\begin{tabular}{|c|c|c|c|}
\hline \multirow{3}{*}{$\begin{array}{l}\text { SIEROTIPI } \\
\text { Gruppo A }\end{array}$} & \multicolumn{2}{|c|}{ frequenza } & \multirow{2}{*}{$\frac{\text { stazioni di isolamento }}{2}$} \\
\hline & S. Koessen & 2 & \\
\hline & S. Nitra & 2 & 1,2 \\
\hline \multirow[t]{12}{*}{ Gruppo B } & S.Agama & 2 & 3 \\
\hline & S. Agona & I & 5 \\
\hline & S. Bredeney & 4 & $4-8$ \\
\hline & S. Derby & 4 & $4-7$ \\
\hline & S. Eppendorf & 1 & I \\
\hline & S. Farsta & 3 & $3-5$ \\
\hline & S. Haifa & 1 & 6 \\
\hline & S. Kingston & 1 & 7 \\
\hline & S. Lagos & 3 & $2-4$ \\
\hline & S. Saintpaul & 2 & $2-4$ \\
\hline & S. Stanley & 4 & $2-6$ \\
\hline & S.Typhimurium & 13 & $3-8$ \\
\hline \multirow[t]{21}{*}{ Gruppo $\mathrm{Cl}-\mathrm{C} 2$} & S. Bonn & I & $\mathrm{I}, 3$ \\
\hline & S. Bovismorbificans & 1 & 4 \\
\hline & S. Brazzaville & 1 & 6 \\
\hline & S. Colorado & 1 & 8 \\
\hline & S. Concord & I & $\mathrm{I}, 3$ \\
\hline & S. Eingedi & 1 & 2 \\
\hline & S. Gabon & 2 & 3 \\
\hline & S. Goldcoast & 1 & 3 \\
\hline & S. Hissar & I & I \\
\hline & S. Kisii & 2 & 2 \\
\hline & S. Kivu & 1 & 4 \\
\hline & S. Koumra & 1 & 5 \\
\hline & S. Kottbus & 3 & $3-7$ \\
\hline & S. Infantis & 5 & $3-6$ \\
\hline & S. Irumu & 1 & 2 \\
\hline & S. Isangi & 1 & 6 \\
\hline & S. Leopoldville & 1 & $5-7$ \\
\hline & S. Montevideo & 2 & $2-3$ \\
\hline & S. Richmond & 1 & I \\
\hline & S. Rissen & 3 & $3-7$ \\
\hline & S. Sandow & 2 & $2-4$ \\
\hline \multirow[t]{2}{*}{ Gruppo D } & S. Berta & I & $1-5$ \\
\hline & S. Enteritidis & 2 & $2-6$ \\
\hline \multirow[t]{4}{*}{ Gruppo EI } & S. Asylanta & I & I \\
\hline & S. Give & 2 & 2 \\
\hline & S. Lekke & I & I \\
\hline & S. Southbank & 1 & 5 \\
\hline Gruppo I & S. Shangani & 1 & 6 \\
\hline \multirow[t]{4}{*}{ Sottospecie II } & Gruppo B & 3 & 8 \\
\hline & Gruppo $\mathrm{Cl}-\mathrm{C} 2$ & 4 & 8 \\
\hline & Gruppo El & & 3 \\
\hline & S. autoagglutinante & 1 & 7 \\
\hline Totale & & 95 & \\
\hline
\end{tabular}

\section{DISCUSSIONE}

Le indagini di controllo svolte nell'area in esame hanno seguito l'andamento delle concentrazioni Salmonella nel corso di un biennio. Le concentrazioni elevate, sintomo di condizioni di alterata qualità igienico-sanitaria ed ambientale delle acque, sono da associare ad immissioni di scarichi non trattati ed a una scarsa funzionalità dei sistemi di trattamento dei reflui urbani ed industriali, 
questi ultimi responsabili anche dell'apporto di sostanze nutritive favorenti la sopravvivenza dei microrganismi nelle acque. Nell'area considerata, inoltre, risulta ancora significativa la presenza di scarichi diretti di origine urbana che immettono nei corpi idrici acque contaminate e fecalizzate.

È comunque da osservare che lo studio svolto ha fornito risultati, almeno nel secondo anno di studio, che poco si discostano da quelli ottenuti da indagini condotte sulla qualità di altri corsi d'acqua italiani. È il caso, ad esempio, del fiume Tevere (3), dove, analogamente a quanto si osserva per l'indagine qui presentata, il grado di contaminazione del fiume, le cui acque presentavano un elevato carico organico, è facilmente riconducibile, non solo a ridotto funzionamento degli impianti di trattamento dei reflui, ma anche alla possibilità di immissioni puntuali non controllate lungo il suo corso.

Sembrerebbe inoltre evidente l'esistenza di una correlazione diretta tra qualità delle acque superficiali e densità abitativa, grado di industrializzazione del territorio e diffusione di pratiche agricole intensive e zootecniche. Infatti, inadeguatezza dei processi di depurazione dei reflui e presenza di animali possono rappresentare una fonte di diffusione permanente del patogeno nell'ambiente. Inoltre, la sua presenza costante in tutte le stazioni di campionamento, anche in concentrazioni elevate, dimostra la sua elevata capacità di sopravvivenza nelle acque.

Non meno interessante è l'osservazione che i sierotipi individuati con maggiore prevalenza nelle acque indagate corrispondono, con rapporti similari, a quelli, più in generale, diffusi nell'ambiente in Italia $(2,7,9,10)$. D'altra parte, gli stessi sierotipi sono riconducibili ai più frequenti isolati umani segnalati sul territorio dal sistema di sorveglianza sulle salmonelle $(8,14)$.

Tra i diversi sierotipi individuati, S. Typhimurium, comunemente isolata da animali ed alimenti, ha la maggiore rilevanza clinica e comunque una grande capacità di adattamento e sopravvivenza all'ambiente acquatico. Di interesse appare anche la grande variabilità di sierotipi identificati, aspetto che può essere legato all'esistenza di diversi serbatoi naturali, alla presenza di animali selvatici e di allevamento e alla loro diversa capacità di crescita e mantenimento nell'ambiente (18). A conferma dell'ampia circolazione di Salmonella nei corpi idrici del bacino del Sarno è stato anche evidenziato che, in uno stesso sito di prelievo, è stato isolato più di un sierotipo.

\section{CONCLUSIONI}

La presenza nelle acque esaminate di diversi sierotipi di Salmonella, legata a situazioni endemi- che locali e territoriali, può rappresentare una condizione di rischio per le popolazioni e un indiscusso serbatoio del patogeno anche in relazione alle sue elevate concentrazioni e capacità di sopravvivenza nell'ambiente.

Dai risultati ottenuti, emerge l'importanza dell'estensione, sul territorio interessato, della rete di sorveglianza ambientale, che potrebbe garantire una migliore attività di prevenzione e la disponibilità di dati più accurati consentendo di valutare meglio il ruolo che l'ambiente gioca nella trasmissione dei patogeni enterici all'uomo.

Sorveglianza epidemiologica, attività di risanamento del territorio, con la scelta di opportuni processi di trattamento delle acque, nonché l'applicazione di adeguati criteri di qualità delle acque, accompagnati da interventi di gestione integrata e da una politica volta al ripristino della funzionalità naturale dell'intero reticolo idrografico, potrebbero rappresentare una soluzione funzionale al miglioramento delle condizioni di qualità igienico-sanitaria delle acque dei corpi idrici del bacino del Sarno e di tutta l'area costiera interessata dalla foce del fiume.

\section{BIBLIOGRAFIA}

1. APAT-IRSA CNR. Manuale dei Metodi Analitici 3. Manuali e Linee Guida. Roma: APAT, 2003: 1153.

2. Bacchi M, Cirillo G, Ranieri M. Enter-net, la rete di sorveglianza delle infezioni da Salmonella e coliformi. ARPA- Rivista 2004; 5: 34-5.

3. Bonadonna L, Briancesco R, Donia D, Ottaviani M, Veschetti E, Divizia M. Aspetti igienico-sanitari delle acque del fiume Tevere: presenza di protozoi patogeni e rapporti di correlazione con parametri microbiologici e chimico-fisici. Ann Ig 2004; 16: 273-80.

4. Brackelsberg CA, Nolan LK, Brown J. Characterization of Salmonella Dublin and Salmonella typhimurium (Copenhagen) isolates from cattle. Vet Res Comm, 1997; 21: 409-20.

5. Centre for Reference and Research on Salmonella. Antigenic formulae of Salmonella. Paris: WHO International Salmonella Centre, Institute Pasteur, 1980.

6. Centro di Referenza Nazionale per le Salmonellosi. Programma Enter-Vet, Sorveglianza delle salmonellosi in ambito veterinario, Istituto Zooprofilattico Sperimentale delle Venezie. Rapporto annuale 2004, in www.izsvenezie.it/dnn/Portals/0/salmonellosi/Report 2004.pdf

7. Cirillo G, Ortali F. Il Centro di riferimento per la tipizzazione di Salmonella spp in EmiliaRomagna. Microbiologia Medica 2006; 21: 16-20.

8. Cirillo G. Evoluzione epidemiologica del genus Salmonella: 25 anni di osservazioni sul territorio di Forlì. Atti RER e Prov.di Ferrara 1986; 63-6.

9. Cirillo G. La sorveglianza delle salmonellosi legate all'ambiente. Atti ISS Roma 2005; 29-30.

10. Cirillo G. Sistema di sorveglianza Enter-net: isolamenti di Salmonella da fonti ambientali. Atti ISS, Roma, 2003.

11. Fisher IST. International trends in Salmonella serotypes 1998-2003 - a surveillance report from the Enter- 
net international surveillance network. Eur Surv 2004; 9: 11-3.

12. Fisher IST. The Enter-net international surveillance network - how it works. Eur Surv 1999; 4: 52-5.

13. Galetta P, Luzzi I, Filetici E, et al. Enter-net: sorveglianza delle infezioni da patogeni enterici. Notiziario dell'Istituto Superiore di Sanità 2005; 18 (7-8): 3-8.

14. Graziani C, Galetta P, Busani L, et al. Infezioni da Salmonella: diagnostica, epidemiologia e sorveglianza. Rapporti Istisan 05/27. Roma: Istituto Superiore di Sanità, 2005.

15. Istituto Nazionale di Statistica (ISTAT). La mortalità per causa delle regioni italiane. Roma, 1997.

16. Lemarchand K, Lebaron P. Influence of mutation frequency on the persistence of Salmonella Enterica serotypes in natural waters. FEMS Microbiol Ecol 2002; 41: 125-31.

17. Melloul AA, Hassani L, Rafouk L. Salmonella contamination of vegetables irrigated with untreated wastewater. World J Microbiol Biotechnol 2001; 17: 207-9.

18. Polo F, Figueras MJ, Inza I, Sala J, Fleisher JM, Guardo J. Prevalence of Salmonella serotypes in environmental waters and their relationships with indicator organisms. Antonie van Leeuwenhoek 1999; 75: 285-92.

19. Popoff MY, Bockermuhl J, Brenner FW. Supplement 1997 (n. 41) to the Kauffmann-White scheme. Res Microbiol 1998; 149: 601-4.

20. Veling J, Barkema HW, van der Schans J, van Zijderveld F, Verhoeff J. Herd-level diagnosis for Salmonella enterica subsp. enterica serovar Dublin infection in bovine dairy herds. Prev Vet Med 2002; 53 (1-2): 31-42.

\section{Lucia Bonadonna}

Istituto Superiore di Sanità

Viale Regina Elena 299 - 00161 Roma

Tel.: 0649902317

E-mail: lucybond@iss.it 